\title{
Third order elastic constants of hexagonal boron nitride
}

\author{
C S MENON \\ Department of Physics, University of Calicut, Calicut 673635, India \\ MS received 8 April 1985; revised 30 May 1985
}

\begin{abstract}
Third order elastic constants of hexagonal Boron Nitride have been evaluated using the Lannard-Jones potential. The results obtained are presented and compared with the only available measurement of $C_{333}$ for this material.
\end{abstract}

Keywords. Third order elastic constants; hexagonal boron nitride; Lannard-Jones potential.

The complete third order elastic constants of hexagonal boron nitride have been obtained using the measured second order elastic constants and their pressure derivatives. The results have been compared with available experimental results.

The potential energy is expanded in powers of the changes in squares of the vector distances $R(I)$ and $R(J)$

$$
\begin{aligned}
\Phi=\Phi_{0} & +O^{\prime} \phi(r)\left(\sum_{I} \Delta R^{2}(I)+\sum_{J} \Delta R^{2}(J)\right)+O^{\prime \prime} \phi(r) \frac{1}{2}\left(\sum_{I}\left(\Delta R^{2}(I)\right)^{2}\right. \\
& \left.+\sum_{J}\left(\Delta R^{2}(J)\right)^{2}\right)+O^{\prime \prime \prime} \phi(r) \frac{1}{6}\left(\sum_{I}\left(\Delta R^{2}(I)\right)^{3}+\sum_{J}\left(\Delta R^{2}(J)\right)^{3}\right),
\end{aligned}
$$

where $I$ refers to the six nearest neighbours of boron nitride of the same type and $J$ to the six neighbours of different type. The operators:

$$
O^{\prime}=\frac{\partial}{\partial r^{2}}, O^{\prime \prime}=O^{\prime 2} \text { and } O^{\prime \prime \prime}=O^{\prime 3}
$$

Using the theory of deformation, the strained lattice vectors are obtained as

$$
\left.\begin{array}{rl}
R_{i}^{\prime}(I)= & R_{i}(I)+\sum_{j} \varepsilon_{i j} R_{j}(I) \\
R_{i}^{\prime}(J)= & R_{i}(J)+\sum_{j} \varepsilon_{i j} R_{j}(J)+W_{i}
\end{array}\right\},
$$

correct to the third order in the deformations. The Lagrangian strain parameters $\eta_{i j}$ are defined by

$$
\eta_{i j}=\frac{1}{2}\left(\varepsilon_{i j}+\varepsilon_{j i}+\sum_{k} \varepsilon_{k i} \varepsilon_{k j}\right),
$$


We define:

$$
\bar{W}_{i}=W_{i}+\sum_{j} \varepsilon_{j i} W_{j}
$$

In terms of these quantities the energy is invariant towards rigid rotations of the crystal. For $K=I, \bar{W}=0$. Substituting for powers of $\Delta R^{2}(K)$ in (1) from (4) and retaining only terms up to the third order in the deformation, one obtains the expression for the strain energy per unit volume in the undeformed state.

The internal displacement $\bar{W}_{i}$ can be obtained in terms of the Lagrangian strain parameters by minimising the strain energy with respect to $\bar{W}_{i}$. It has been shown by Srinivasan (1966) that to get the strain energy correct to the third order in strain it is enough if we get the internal displacement up to the first order in strain.

$$
\bar{W}_{i}=-\sum_{j, k} \eta_{j k}\left(\sum_{j} R_{i} R_{j} R_{k}\right) / \sum_{j} R_{i}^{2}
$$

making the lattice sums

$$
\bar{W}_{x}=\left[D / 2(3)^{1 / 2}\right]\left(\eta_{x x}-\eta_{y y}\right) \text {, }
$$

and

$$
\bar{W}_{y}=-\left[D / 2(3)^{1 / 2}\right]\left(\eta_{x y}+\eta_{y x}\right) \text {. }
$$

Substituting for $\bar{W}_{i}$ in the expression for the strain energy per unit volume in the undeformed state and comparing the resulting expression with the expression for elastic theory energy density,

$$
U=\frac{1}{2} \sum_{i j, k l} C_{i j, k i} \eta_{i j} \eta_{k i}+\frac{1}{6} \sum_{i j, k l, m n} C_{i j, k l, m n} \eta_{i j} \eta_{k i} \eta_{m n} .
$$

we get the expression for the second order and third order elastic constants as:

$$
\begin{array}{ll}
C_{11}=\frac{29}{3} P, C_{12}=\frac{11}{3} P, C_{44}=\frac{8}{3} P, C_{33}=\frac{32}{3} P, C_{13}=\frac{8}{3} P \\
C_{111}=\frac{247}{18} Q+\frac{P}{3}, & C_{222}=\frac{301}{18} Q+\frac{P}{3} \\
C_{333}=\frac{256}{18} Q, & C_{112}=\frac{85}{18} Q-\frac{1}{3} P \\
C_{113}=\frac{16}{18} Q+\frac{4}{3} P, & C_{123}=\frac{16}{18} Q-\frac{4}{3} P \\
C_{133}=\frac{64}{18} Q, & C_{144}=\frac{16}{18} Q \\
C_{155}=\frac{16}{18} Q, & C_{344}=\frac{64}{18} Q \\
\text { where } P=\frac{K_{2} D^{4}}{V_{z}}, & Q_{2}=\frac{K_{3}}{V_{z}} D^{6} ; \\
K_{2}=O^{\prime \prime} \Phi, & K_{3}=O^{\prime \prime \prime} \Phi ;
\end{array}
$$


Table 1. Values of second order elastic constants (in $10^{11} \mathrm{dyn} / \mathrm{cm}^{2}$ ) and pressure derivatives of the elastic constants of hexagonal boron nitride.

\begin{tabular}{lcccccc}
\hline \multicolumn{1}{c}{$C_{i j}$} & $C_{11}$ & $C_{33}$ & $C_{44}$ & $C_{66}$ & $C_{12}$ & $C_{13}$ \\
\hline Present work & 2.93 & 3.24 & 0.81 & 0.91 & 1.11 & 0.81 \\
$\begin{array}{l}\text { Experiment } \\
\text { (Green et al 1976) }\end{array}$ & - & $3.24 \pm 0.3$ & - & - & - & - \\
Pressure derivative & 11.63 & 12.90 & 2.70 & 3.16 & 5.31 & 3.69 \\
\hline
\end{tabular}

Table 2. Values of third order elastic constants (in $10^{11} \mathrm{dyn} / \mathrm{cm}^{2}$ ) of hexagonal boron nitride

\begin{tabular}{lcccccccccc}
\hline \multicolumn{1}{c}{$C_{i j k}$} & $C_{111}$ & $C_{112}$ & $C_{113}$ & $C_{123}$ & $C_{133}$ & $C_{344}$ & $C_{333}$ & $C_{222}$ & $C_{144}$ & $C_{155}$ \\
\hline Present work & -49.8 & -17.3 & -2.8 & -3.6 & -12.9 & -12.9 & -51.7 & -60.7 & -3.2 & -3.2 \\
$\begin{array}{l}\text { Experiment } \\
\text { (Green et al 1976) }\end{array}$ & - & - & - & - & - & - & $-58.96 \pm 2$ & - & - & - \\
\hline
\end{tabular}

$D$ is the nearest neighbour distance and $V_{z}$ is the volume of the primitive cell. Constraints like $C_{13}=C_{44}$ occur because of the assumption that the forces involved are purely central. The pressure derivatives of the acoustic wave velocities for various crystal systems are given by Brugger (1965). Assuming a potential energy form of the Lannard-Jones type,

$$
\Phi(r)=-\frac{A}{r^{6}}+\frac{B}{r^{12}} .
$$

the second and third order elastic constants have been obtained from the set of expression (11) and (12) respectively.

Table 1 gives the second order elastic constants of hexagonal boron nitride along with their pressure derivatives. Table 2 reports the ten third order elastic constants of hexagonal boron nitride together with the only available measured value of $\mathrm{C}_{333}$ (Green et al 1976).

This work is the first step in obtaining the mode Gruneisen Gammas and the thermal expansion of boron nitride at low temperatures.

\section{References}

Brugger K 1965 J. Appl. Phys. 36768

Green J F, Bolland T K and Bolland J W 1976 J. Chem. Phys. 64656

Srinivasan R 1966 Phys. Rev. 144620 